\title{
On the Relationship between Water Withdrawal and Income: Smooth Transition Regression (STR) Approach
}

\author{
Mohsen Mehrara ${ }^{1}$, Abdolnaser Hemati ${ }^{1}$, Ali Sayehmiri ${ }^{1,2}$ \\ ${ }^{1}$ Department of Economics, Faculty of Economics, University of Tehran, Tehran, Iran \\ ${ }^{2}$ Department of Economics, Faculty of Humanities, Ilam University, Ilam, Iran \\ E-mail:mmehrara@ut.ac.ir,a.hemmati@ut.ac.ir,alisayehmiri@ut.ac.ir \\ Received July 2, 2011; revised August 11, 2011; accepted August 22, 2011
}

\begin{abstract}
The Purpose of this paper is to investigate the relationship between annual water withdrawal (AWW) and income in some countries. To achieve this end, a smooth transition regression (STR) model on cross section data of 163 countries in 2006 was used. The findings support the nonlinearity of the link between AWW and income. The findings, further, reveal the income elasticity of AWW as a bible-shaped curve. Hence, the policies and management processes in water sector including water allocation between activities and reigns should take into account the development degree and also focus on income level, water scarcity and the economic, social and ecological structure in each country.
\end{abstract}

Keywords: Water-Income, STR, NRBEKC, Elasticity, Socio-Economic Structure

\section{Introduction}

Freshwater resources are vital for maintaining human life, health, agricultural production, economic activities as well as critical ecosystem functions. As populations and economies grow, new constraints on freshwater resources are appearing, raising problems for limits of water availability. Accordingly, the analysis of the national water withdrawal intensity measurement becomes an important policy issue. To serve these purposes, some water withdrawal efficiency indicators have been developed and applied to explain differences in performance between countries and international benchmarking [1]. It should be noted that the income elasticity of water withdrawal is one solution used in this paper.

In recent years, the relationship between income elasticity of natural resources use and income has attracted an increasing attention among academic, non-governmental organizations, and the media. A notable empirical finding of the recent environmental economics literature has been the existence of an inverted U-shaped relationship between per capita income and pollution (per capita emissions) of many local air pollutants [2]. Since this relationship bears a resemblance to the Kuznets relationship between income and income inequality, it is known as the Environmental Kuznets Curve (EKC) and has spawned a vast number of papers in recent years. In addition, attempts have been made to estimate EKCs for a wide range of environmental indicators, including energy use, deforestation and municipal waste [3-7].

The shape of the EKC, attributed to scale, composition and technique effects (SCTE) as discussed below, would also seem to apply to (income elasticity of) water consumption. The main reason to disregard water use in EKC studies would appear to be a lack of socioeconomic-hydrological data, although some recent investigations and dataset have now resolved somewhat this problem [8-12].

In this paper, we examine the relationship between AWW per capita and GDP per capita using Smooth Transition Regression (STR) model for 163 countries across the world based on cross section data in 2006. The following section will provide a brief review of the related literature. Section 3 introduces the econometric methodology and empirical results, and the final section presents the conclusions of the present study.

\section{Natural Resources Based on Environmental Kuznets Curve (NRBEKC)}

The majority of EKC literature examines pollution levels as a function of income. This has led to the criticism that such research ignores the natural resource component of 
environmental quality [2,13-16]. These studies tend to treat resource use identical to pollution as an indicator of environmental quality pointing to natural resources based on environmental Kuznets curve (NRBEKC). Like pollution, resource use can provide an economic benefit coupled with an undesired environmental impact. Thus, many of the theoretical explanations for the existence of EKCs for natural resources mirror those for pollution.

The inverted U relationship between income and pollution is typically explained in terms of the interaction of scale, composition and technique effects (SCTEs). The scale effect (SE) implies that as the scale of the economy grows (ceteris paribus), AWW will do so. The composition effect (CE), however, refers to the fact that as economies develop, there is totally a change in emphasis from heavy industry to light manufactures and services sectors, and also from high water intensity to low water intensity in industrial, agriculture and domestic sectors. Since the latter are typically less resource intensive than the former, the composition effect of growth, ceteris paribus, will reduce water use. Finally, there is the technique effect (TE). As incomes rise there is likely to be an increased demand for environmental regulations [5]. The effect of these regulations must be considered to reduce water intensity due to improved techniques of production and consumption.

\section{Methodology}

\subsection{Smooth Transition Regression (STR)}

The smooth transition regression (STR) model is a nonlinear regression model that may be viewed as a further development of the switching regression model introduced by [17]. The STR model originated as a generalization of a particular switching regression model in the work of [17]. These authors considered two regression lines and devised a model in which the transition from one line to the other is smooth. The earliest references in the econometrics literature are $[18,19]$. Recent accounts include [20-25]. The standard STR model is defined as follows:

$$
\begin{aligned}
y_{t} & =\varphi^{\prime} z_{t}+\theta^{\prime} z_{t} G\left(\gamma, c, s_{t}\right)+u_{t} \\
& =\left\{\varphi+\theta G\left(\gamma, c, s_{t}\right)\right\}^{\prime_{t}}+u_{t}, \quad t=1, \cdots, T,
\end{aligned}
$$

where $\mathbf{z}_{t}^{\prime}=\left(\mathbf{w}_{t}^{\prime}, \mathbf{x}_{t}^{\prime}\right)^{\prime}$ is a veçtor of explanatory variables $_{t}^{\prime}=\left(1, y_{t-1}, \cdots, y_{t-p}\right)^{\prime}$ and $\mathbf{x}_{t}^{\prime}=\left(x_{1 t}, \cdots, x_{k t}\right)^{\prime}$ are a vector of exogenous variables. Furthermore, $\varphi=\left(\varphi_{0}\right.$, $\left.\varphi_{1}, \cdots, \varphi_{m}\right)^{\prime}$ and $\theta=\left(\theta_{0}, \theta_{1}, \cdots, \theta_{m}\right)^{\prime}$ are $((m+1) \times 1)$ parameter vectors and $u_{t} \sim \operatorname{iid}\left(0, \sigma^{2}\right)$ are given. Transition function $G\left(\gamma, c, s_{t}\right)$ is a bounded function of the continuous transition variable $s_{t}$, continuous everywhere in the parameter space for any value of $s_{t}, \gamma$ is the slope parameter and $c=\left(c_{1}, \cdots, c_{K}\right)^{\prime}$ which is a vector of location parameters, $c_{1} \leq \cdots \leq c_{K}$. The last expression in Equation (1) indicates that the model can be interpreted as a linear model with stochastic time-varying coefficients $\varphi+\theta G$ $\left(\gamma, c, s_{t}\right)$. In this paper it is assumed that the transition function is a general logistic function:

$$
G\left(\gamma, c, s_{t}\right)=\left(1+\exp \left\{-\gamma \prod_{t=1}^{t}\left(s_{t}-k_{t}\right)\right\}\right)^{-1}
$$

where $\gamma>0$ is an identifying restriction. Equations (1) and (2) jointly define the logistic STR (LSTR) model. The most common choices for $K$ are $K=1$ and $K=2$. For $K=1$, the parameters $\varphi+\theta G\left(\gamma, c, s_{t}\right)$ change monotonically as a function of $s_{t}$ from $\varphi$ to $\varphi+\theta$. For $K=2$, they change symmetrically around the midpoint $\left(c_{1}+c_{2}\right) /$ 2 , where this logistic function attains its minimum value. The minimum lies between zero and $1 / 2$. It reaches zero when $\gamma \rightarrow \infty$ and equals $1 / 2$ when $c_{1}=c_{2}$ and $\gamma<\infty$. Slope parameter $\gamma$ controls the slope and $c_{1}$ and $c_{2}$ the location of the transition function. Transition function (2) with $K=1$ is also the one that [18] proposed, whereas [18] and Chan \& Tong (1986) favored the cumulative distribution function of a normal random variable. In fact, these two functions are close substitutes.

The LSTR model with $K=1$ (LSTR1 model) is capable of characterizing asymmetric behavior. As an example, it is supposed that $s_{t}$ measures the phase of the business cycle. Then the LSTR1 model can describe processes whose dynamic properties are different in expansions from what they are in recessions, and the transition from one extreme regime to the other is smooth. On the other hand, the LSTR2 model $(K=2)$ is appropriate in situations in which the local dynamic behavior of the process is similar at both large and small values of $s_{t}$ and different in the middle (For further work on parameterizing the transition in the STR framework, see [18]. When $\gamma=0$, the transition function $G\left(\gamma, c, s_{t}\right) \equiv 1 / 2$, and thus the STR model (1) nests the linear model. At the other ends, when $\gamma \rightarrow \infty$, the LSTR1 model approaches the switching regression model with two regimes that have equal variances. When $\gamma \rightarrow \infty$ in the LSTR2 model, the result is another switching regression model with three regimes in which the outer regimes are identical and the mid regime is different from the other two. It is noteworthy that an alternative to the LSTR2 model exists, the so-called exponential STR (ESTR) model. It is Equation (1) with the follow transition function:

$$
G_{E}\left(\gamma, c, s_{t}\right)=1-\exp \left\{-\gamma\left(s_{t}-c_{t}^{*}\right)^{2}\right\}
$$

This function is symmetric around $s_{t}=c_{1}^{*}$ and has, at low and moderate values of slope parameter $\gamma$, approxi- 
mately the same shape, albeit a different minimum value (zero), as (2). Because this function contains one parameter less than the LSTR2 model, it can be regarded as a useful alternative to the corresponding logistic transition function. It has a drawback, however. When $\gamma \rightarrow \infty$, (1) becomes practically linear with (3), for the transition function equals zero at $s_{t}=c_{1}^{*}$ and unity elsewhere. The ESTR model is therefore not a good approximation to the STR2 model when $\gamma$ in the latter is large and $c_{2}-c_{1}$ is at the same time not close to zero. In practice, the transition variable $s_{t}$ is a stochastic variable and very often an element of $\mathbf{z}_{t}$. It can also be a linear combination of several variables. In some cases, it can be a difference of an element of $\mathbf{z}_{t}$; see [18] for a univariate example. A special case, $s_{t}=t$, yields a linear model with deterministically changing parameters. When $\mathbf{x}_{t}$ is absent from (1) and $s_{t}=y_{t-d}$ or $s_{t}=\Delta y_{t-d}, d>0$, the STR model becomes a univariate smooth transition autoregressive model [19] for more discussion).

\subsection{The Modeling Cycle}

In this section we consider modeling nonlinear relationships using STR model (1) with transition function (2). We present a modeling cycle consisting of three stages: specification, estimation, and evaluation. The specification stage entails two phases. First, the linear model forming the starting point is subjected to linearity tests, and then the type of STR model (LSTR1 or LSTR2) is selected. Economic theory may give an idea of which variables should be included in the linear model but may not be particularly helpful in specifying the dynamic structure of the model. Linearity is tested against an STR model with a predetermined transition variable. If economic theory is not explicit about this variable, the test is repeated for each variable in the predetermined set of potential transition variables, which is usually a subset of the elements in $\mathbf{z}_{t}$. Testing linearity against STAR or STR has been discussed, for example, in [20,21].

The resulting test is more powerful than both the LSTR1 $(K=1)$ and LSTR2 $(K=2)$ models. Assume now that the transition variable $s_{t}$ is an element in $\mathbf{z}_{t}$ and let $\mathbf{z}_{t}=\left(1, \mathbf{z}_{t}^{\prime}\right)^{\prime}$, where $\mathbf{z}_{t}^{\prime}$ is an $(m \times 1)$ vector. The approximation yields, after merging terms and parameterizing, the following auxiliary regression:

$$
y_{t}=\beta_{0}^{\prime} x_{t}+\sum_{j=1}^{t} \beta_{j}^{\prime} \tilde{x}_{t} s_{t}^{j}+u_{t}^{*}, \quad t=1, \cdots, T
$$

where $u_{t}^{*}=u_{t}+R_{3}\left(\gamma, c, s_{t}\right) \theta \mathbf{z}_{t}^{\prime}$ with the remainder $R_{3}(\gamma$, $\left.c, s_{t}\right)$. The null hypothesis is $\mathrm{H} 0: \beta_{1}=\beta_{2}=\beta_{3}=0$ because each $\beta j, j=1,2,3$, is of the form $\gamma \tilde{\beta} j$, where, $\tilde{\beta} j \neq 0$ is a function of $\theta$ and $c$. This is a linear hypothesis in a linear (in parameters) model. Because $u_{t}^{*}=u_{t}$ under the null hypothesis, the asymptotic distribution theory is not affected if an LM-type test is used.

$$
G\left(\gamma, c, s_{t}\right)=\left(1+\exp \left\{-\left(\gamma / \hat{\sigma}_{s}^{k}\right) \prod_{k=1}^{K}\left(s_{t}-c_{k}\right)\right\}\right)^{-1}, \gamma>0
$$

\section{Empirical Results}

The basis of our empirical approach is exactly the same as that used by many authors in literature. The observations in this modeling experiment come from AQUSTAT FAO and WDI database of the [26,27]. The purpose of the study is to investigate the effect of GDP per capita on the annual water withdrawal (AWW). The AWW is assumed to be a nonlinear function of the GDP per capita. Figure 1 demonstrates a clearly nonlinear relationship between the logarithmic values of GDP per capita ( $x$-axis) and the AWW ( $y$-axis) as long as kernel fitting curve.

The sample consisted of annual water withdrawal (AWW) per capita in cubic meter and GDP per capita in 2000 constant dollar for 163 countries of the world. Fitting a linear model to the data yields:

$$
\begin{gathered}
L(\boldsymbol{Y i})=\underset{(2.356)}{1.644}+\underset{(5.671)}{0.460 \times} L(X i)+\tilde{\varepsilon}_{t} \\
\tilde{\sigma}_{y}=1.248, n=163, \quad R^{2}=0.166, \\
p^{\text {RESET }}(1,160)=10.27
\end{gathered}
$$

An STR model is fitted to the logarithmic data. The transition function is defined as a logistic function. Where $\tilde{\sigma}_{y}$ equals the residual standard deviation and $p^{\text {RESET }}$ is the p-value of the RESET test. The test does indicate serious misspecification of (6). On the other hand, the residuals arranged according to $\boldsymbol{L} \boldsymbol{X}_{i}$ in ascending order and graphed in Figure 2 show that the linear model is not adequate. It can be seen in $p^{\text {RESET }}$ large value in Equation (6).

The results of the linearity tests appearing in Table 1 p-values are remarkably small. Hypothesis $\mathrm{H}_{0}$ is the gen-

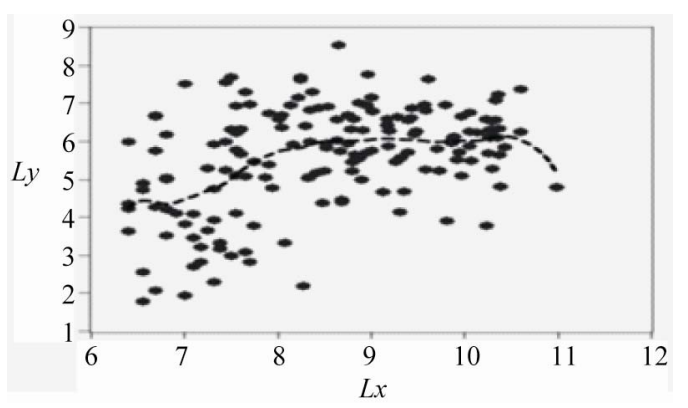

Figure 1. Observations of the logarithmic $x$-axis, and the logarithm of the $y$-axis. 


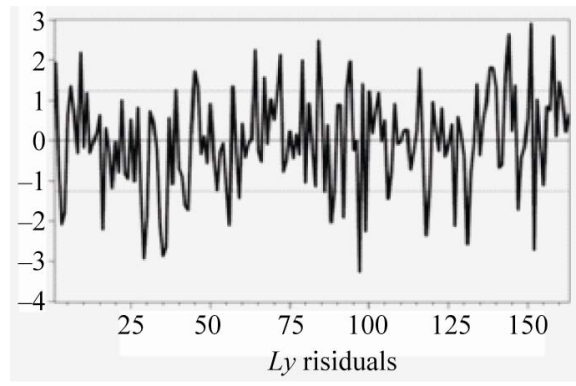

Figure 2. Residuals of (1) (x-axis: the value of $x_{i}$; $y$-axis: residual $\varepsilon_{t}$ ).

Table 1. P-values of the linearity tests of model (6).

\begin{tabular}{lr}
\hline Hypothesis & p-Value \\
\hline $\mathrm{H}_{0}$ & $9.2627 \mathrm{e}-03$ \\
$\mathrm{H}_{4}$ & $2.3688 \mathrm{e}-01$ \\
$\mathrm{H}_{3}$ & $6.2594 \mathrm{e}-01$ \\
$\mathrm{H}_{2}$ & $1.6281 \mathrm{e}-03$ \\
\hline
\end{tabular}

eral null hypothesis based on the third-order Taylor expansion of the transition function. Hypotheses $\mathrm{H}_{04}, \mathrm{H}_{03}$, and $\mathrm{H}_{02}$ are the ones discussed in the Section of methodology. Because the p-value of the test of $\mathrm{H}_{03}$ is much larger than the ones corresponding to testing $\mathrm{H}_{04}$ and $\mathrm{H}_{02}$.

The choice of $K=1$ in Equation (7) (the LSTR1 model) is quite clear. This is also obvious from Figure 1, for there appears to be a single transition from one regression line to the other. The next step is to estimate the LSTR1 model, which yields:

$$
\begin{gathered}
L y_{i}=-\underset{(4.373)}{9.560-\underset{(0.634)}{0.7746} L X_{i}}-\left(\underset{(4.483)}{\left.4.763-\underset{(0.643)}{0.901} L X_{i}\right)}\right. \\
{\left[1+\exp (\underset{(1881.057)}{-332.387} / \hat{\sigma} x)\left(L X_{i}-\underset{(0.1704)}{7.4068}\right)\right]^{-1}} \\
T=163, R^{2}=2.7975 \mathrm{e}-01, \sigma=1.11754, \sigma x=1.2086, \\
\sigma / \sigma_{L}=0.971
\end{gathered}
$$

where, $\sigma_{l x}$ is the sample standard deviation of $L x_{i}, \sigma$ is the residual standard deviation of linear model and $\sigma_{L}$ is that of non-linear one. It should be noted that there are two large standard deviations, which suggests that the full model may be somewhat over parameterized. This is often the case when the STR model is based on the linear model without any restrictions. Model (7) is an example of such a situation. It may appear strange that the need to reduce the size of the model is obvious in this model already because it only has a single explanatory variable.

The first reaction of the model would perhaps be to tighten the specification by removing the nonlinear intercept, Restriction $\varphi=0$ or $G\left(L x_{i}, \gamma, c\right)=0$. Another possibility would be to restrict the intercepts by imposing the other exclusion restriction $\varphi_{0}=\theta_{0}$. In fact, the first alternative yields a model with a slightly better fit than the latter one. The model estimated with this restriction is:

$$
\begin{gathered}
L y_{i}=\underset{(0.032)}{0.627} L X_{i}+\left(\underset{(1.303)}{4.696-0.490} L X_{i}\right) \\
\left.\left[1+\exp (\underset{(28.223)}{-19.765 / \widehat{\sigma L} x})\left(L X_{i}-7.465\right)\right]_{(0.129)}\right]^{-1} \\
T=163, R^{2}=2.3839 \mathrm{e}-01, \sigma=1.205, \sigma \mathrm{Lx}=1.209, \\
\frac{\sigma}{\sigma L x}=0.997
\end{gathered}
$$

The estimated standard deviations of all estimates in (7) are now appreciably small, and thus further reduction of the model size is not necessary. The fit of both (7) and (8) is vastly superior to that of (6), whereas there is little difference between the two LSTR1 models. The residual standard deviation of these models is only about onetenth of the corresponding figure for (2). Such an improvement is unthinkable when economic time series are being modeled. The graph of the transition function as a function of the observations in Figure 3 shows that the transition is indeed smooth.

The test of no additive nonlinearity $\left[\mathrm{H}_{0}: \beta_{1}=\beta_{2}=\beta_{3}=\right.$ 0 in (transition function)] has the $p$-value of 0.0010 . In testing $\left[\mathrm{H}_{02}: \beta_{1}=0 \mid \beta_{2}=\beta_{3}=0\right.$, a test based on a first order Taylor expansion of $\left.\mathrm{H}\left(\gamma_{2}, c_{2}, x_{2 \mathrm{i}}\right)\right]$ and thus one against another LSTR1component, we find that the $p$ value of the test equals 0.017 . These results show that nonlinearity in this data set has been adequately characterized by the LSTR1 model. The tests of no error autocorrelation and parameter constancy are not meaningful here in the same way as they are in connection with time series models, and they have therefore not been applied to model (8).

We modify this approach by using STR model recently developed by Gonzalez et al. [28].

$$
L y_{i}=\alpha_{i}+\beta_{0} L x_{i}+\beta_{1} L x_{i} \cdot G\left(L x_{i}, \gamma, c\right)+\varepsilon_{i}
$$

where $\varepsilon_{i}$ is i.i.d $\left(0, \sigma_{x}^{2}\right)$ and the transition function $G$ is:

$$
G\left(L x_{i}, \gamma, c\right)=\frac{1}{1+\exp \left(-\gamma\left(L x_{i}-c\right)\right)}, \quad \gamma>0,
$$

\section{Analysis}

According on empirical result the relationship between water and income is nonlinear model so we can calculate the elasticity of water. In STR model, income elasticity of AWW per capita $\left(E_{i}\right)$ depends on (log GDP per pita) level $\left(L x_{i}\right)$. So it allows the parameters to change smoothly as a function of the threshold or transition variable. Indeed, the elasticity of income is explained by 


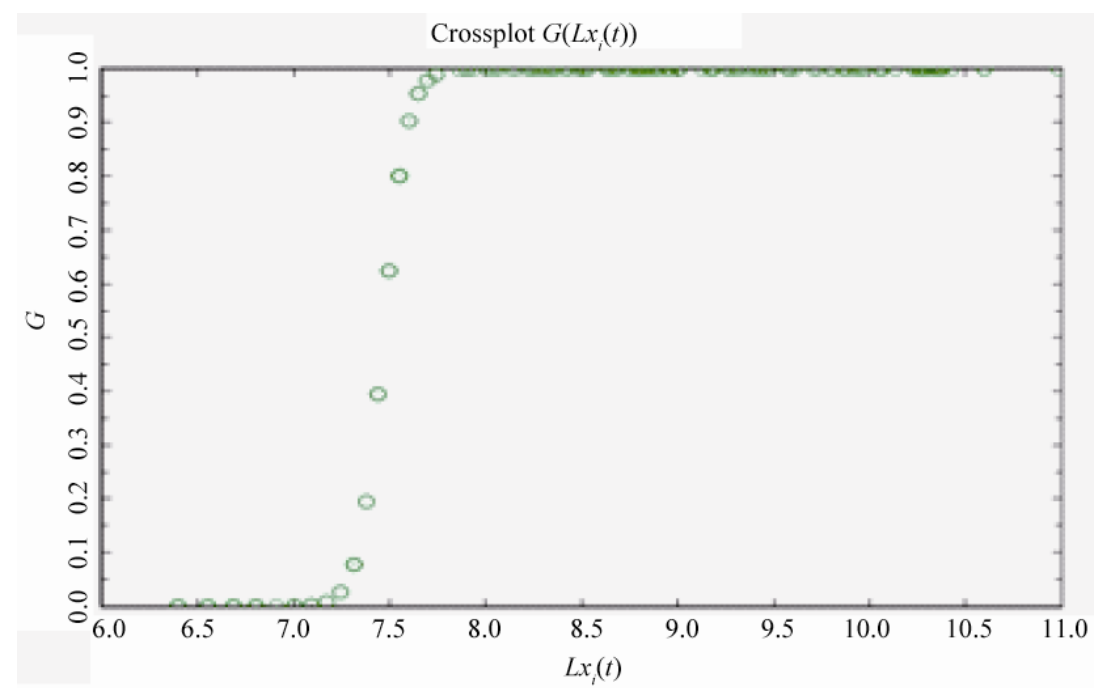

Figure 3. Transition function of model (3) as a function of the transition variable.

the weighted average of parameters including $\beta_{0}$ and $\beta_{1}$. The income elasticity of $\left(\mathrm{E}_{i}\right)$ for country $i_{\mathrm{th}}$ is:

$$
\mathrm{E}_{i}=\frac{\partial L y_{i}}{\partial L x_{i}}=\alpha+\beta_{0} g\left(L x_{i}, \gamma, c\right)+\beta_{1} L x_{i} \frac{\partial g\left(L x_{i} ; \gamma ; c\right)}{\partial L x_{i}},
$$

In this specification, a negative value of $\beta_{1}$ may also lead to the increase of elasticity. The estimated parameters in this part could not be interpreted as elasticity. In Equation (12), the income elasticity of AWW ( $\left.E_{i}\right)$ has been presented. All calculations are computed with Matlab and JMULTI software. The equation used to calculate elasticity is given as the Equation (12).

On the Figure 4, this elasticity is displayed for all pos-

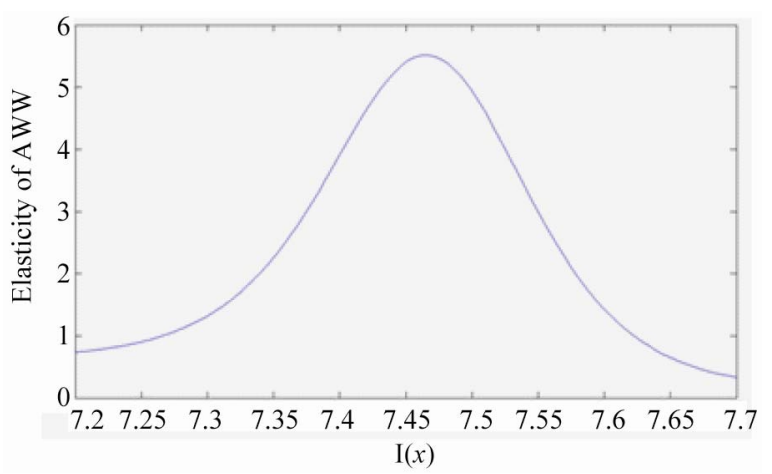

Figure 4. The relationship between GDP per capita and AWW's elasticity. sible values of the transition variable (GDP per capita). Elasticity is increased slightly according to the income level. Moreover, there is strong evidence that the relationship between per capita income and elasticity of AWW is bible-shaped.

\section{Conclusions}

This study reports on the use of a smooth transition regression model based on cross section data to estimate the relationship between AWW and income for 163 countries throughout the world. The findings yielded an inverse U-shaped curve for world countries in the sample data. These results justify ideas of (NRBEKC), (STCE) and (OVW) concepts that are combining ecological and social benefits as a whole. The findings further show that income and socioeconomic criteria along with water scarcity can have an effect on water withdrawal in water-scarce countries and water intensity of use.

The results, also, have important implications for the models of water use and economic growth developed by [29], and the other issues of water-income relationship by $[1,5,8,11]$. This model needs to be modified in a number of ways to account for the water savings accompanying a rapid structural transformation of an economy. This can be accomplished either by estimating the water savings attributable to the structural transformation of an economy or revising the way we think about water use during the transition.

$$
\mathrm{E}_{i}=\frac{\partial L y_{i}}{\partial L x_{i}}=0.62680-\frac{-0.48965}{\left[1+\exp \left(-19.765\left(L x_{i}-7.465\right)\right)\right]}-\frac{19.765\left(0.48965\left(L x_{i}-4.696\right) \times \exp \left(-19.765\left(L x_{i}-7.465\right)\right)\right)}{\left[1+\exp \left(-19.765\left(L x_{i}-7.465\right)\right)\right]^{2}}
$$




\section{Acknowledgements}

This paper was funded by grant from the University of Tehran submitted to the author

\section{References}

[1] P. H. Gleick and M. Palaniappan, "Peak Water Limits to Freshwater Withdrawal and Use," Proceedings of the $\mathrm{Na}$ tional Academy of Sciences, Vol. 107, No. 25, 2010, pp. 11155-11162. doi:10.1073/pnas.1004812107

[2] K. B. Arrow, R. Bolin, P. Costanza, G. M. Grossman and A. B. Krueger, "Economic Growth and the Environment," Quarterly Journal of Economics, Vol. 110, No. 2 1995, pp. 353-377. doi:10.2307/2118443

[3] S. Dinda, "Environmental Kuznets Curve Hypothesis: A Survey," Ecological Economics, Vol. 49, No. 4, 2004, pp. 431- 455. doi:10.1016/j.ecolecon.2004.02.011

[4] A. Camas, P. Ferrao and P. Conceicao, "A New Environmental Kuznets Curve? Relationship between Direct Material Input and Income Per Capita: Evidence from Industrialized Countries," Ecologic Economics, Vol. 46, No. 2, 2003, pp. 217-229. doi:10.1016/S0921-8009(03)00123-X

[5] M. A. Cole, "Trade, the Pollution Haven Hypothesis and Environmental Kuznets Curve: Examining the Linkages," Ecological Economics, Vol. 48, No. 1, 2004, pp. 71-81. doi:10.1016/j.ecolecon.2003.09.007

[6] B. Barbier, "Introduction to the Environmental Kuznets Curve Special Issue," Environmental Development Economics, Vol. 24, 1997, pp. 369-381.

[7] D. I. Stern, M. S. Common and E. B. Barbier, "Economic Growth and Environmental Degradation: The Environmental Kuznets Curve and Sustainable Development," World Development, Vol. 24, No. 7, 1996, pp. 1151-1160. doi:10.1016/0305-750X(96)00032-0

[8] A. Y. Hoekstra, A. K. Chapagain, M. M. Aldaya and M. M. Mekonnen, "Water Footprint Manual: State of the Art," Water Footprint Network, 2009.

[9] A. Y. Hoekstra and A. K. Chapagain, "Water Footprints of Nations: Water Use by People as a Function of Their Consumption Pattern," Water Resources Management, Vol. 21, No. 1, 2007, pp. 35-48. doi:10.1007/s11269-006-9039-x

[10] M. T. Tock, "Freshwater Use, Freshwater Scarcity, and Socioeconomic Development," Journal of Environment and Development, Vol. 7, No. 3, 1998, pp. 278-301. doi:10.1177/107049659800700304

[11] M. T. Tock, "The Dewatering of Economic Growth What Accounts for the Declining Water-Use Intensity of Income?" Journal of Industrial Ecology, Vol. 4, No. 1, 2001, pp. 57-73.

[12] I. A. Shiklomanov, "Appraisal and Assessment of World Water Resources," Water International, Vol. 25, No. 1, 2000, pp. 11-32. doi:10.1080/02508060008686794

[13] N. Shafik and S. Bandyopadhyay, "Economic Growth and Environmental Quality: Time Series and Cross-
Country Evidence," The World Bank, Washington, DC, 1992.

[14] K. E. E. Martinez, J. C. Crenshaw and G. M. Jenkins. "Deforestation and the Environmental Kuznets Curve: A Cross-National Investigation of Intervening Mechanisms," Social Science Quarterly, Vol. 83, No. 1, 2002, pp. 226-243. doi:10.1111/1540-6237.00080

[15] R. J. Culas, "Deforestation and the Environmental Kuznets Curve: An Institutional Perspective," Ecological Economics, Vol. 61, No. 2-3, 2007, pp. 429-437. doi:10.1016/j.ecolecon.2006.03.014

[16] R. E. Quant, "The Estimation of Parameters of a Linear Regression System Obeying Two Separate Regimes," Journal of the American Statistical Association, Vol. 53, 1958, pp. 873-880. doi:10.2307/2281957

[17] D. W. Bacon and D. G. Watts, "Estimating the Transition between Two Intersecting Straight Lines," Biometrika, Vol. 58, No. 3, 1971, pp. 525-534. doi:10.1093/biomet $/ 58.3 .525$

[18] S. M. Goldfield and R. Quant, "Nonlinear Methods in Econometrics," North-Holland, Amsterdam, 1972.

[19] D. S. Maddala, "Econometrics," McGraw-Hill, New York, 1977.

[20] C. W. Granger and T. Terasvirta, "Modeling Nonlinear Economic Relationships," Oxford University Press, Oxford, 1993, Oxford.

[21] T. Terasvirta, "Specification, Estimation and Evaluation of Smooth Transition Autoregressive Models," Journal of the American Statistical Association, Vol. 89, 1994, pp. 208-218. doi: $10.2307 / 2291217$

[22] T. Terasvirta, "Modeling Economic Relationships with Smooth Transition Regressions," Handbook of Applied Economic Statistics, Dekker, New York, 1998, pp. $507-$ 552.

[23] P. H. Franses and D. Vandijk, "Non-Linear Time Series Models in Empirical Finance," Cambridge University Press, Cambridge, 2000. doi:10.1017/CBO9780511754067

[24] D. A. Dickey and W. A. Fuller, "Estimators for Autoregressive Time Series with a Unit Root," Journal of the American Statistical Association, Vol. 74, 1979, pp. $427-$ 431. doi: $10.2307 / 2286348$

[25] K. S. Chan and H. Tong, "On Estimating Thresholds in Autoregressive Models," Journal of Time Series Analysis, Vol. 7, No. 3, 1986, pp. 178-190. doi:10.1111/j.1467-9892.1986.tb00501.x

[26] Food and Agriculture Organization (FAO) of the United Nations, "AQUASTAT Online Database," 2006.

[27] World Bank, "World Development Indicators Online Database," 2007.

[28] A. Gonzalez, T. Terasvirta and D. Van Dijk, "Panel Smooth Transition Regression Model," Working Paper Series in Economics and Finance, No. 604, 2005.

[29] E. B. Barbier, "Water and Economic Growth," Economic Record, Vol. 80, No. 248, 2004, pp. 1-16. doi:10.1111/j.1475-4932.2004.00121.x 